\title{
HOMOGENEIZACIÓN DE LAS SERIES DE TEMPERATURA DEL AIRE EN LA REPÚBLICA CHECA DURANTE EL PERÍODO INSTRUMENTAL*
}

\author{
Štěpánek Petr \\ Department of Geography, Masaryk University, Kǒtlárská 2, 61137 Brno, \\ República Checa \\ E-mail: petr.stepanek@chmi.cz
}

\begin{abstract}
Resumen: En el presente trabajo se muestra el proceso de homogeneización de las series de temperatura del aire mensuales, estacionales y anuales de casi 200 observatorios de la República Checa durante el periodo instrumental desde 1771. El análisis de la homogeneidad relativa de las citadas series se realizó con tres pruebas: el Standard Normal Homogeneity Test (Alexandersson, 1986), el Bivariate Test (Maronna y Yohai, 1978), y el método de Vincent (1996). Las series de referencia se crearon mediante tres métodos distintos, con el fin de poder ser más precisos en la toma de decisiones finales. Todos los análisis realizados en el presente trabajo se han efectuado con software específicamente desarrollado por el autor para el tratamiento automático de datos climáticos, homogeneización, ajustes, etc: AnClim y ProcData. El software AnClim es de libre disposición (http://www.sci.muni.cz/ pest).
\end{abstract}

Palabras Clave: Temperatura. Homogeneización. Programa AnClim. Programa ProcData. República Checa.

\begin{abstract}
This paper deals with homogenization of monthly, seasonal and annual air temperature series of almost 200 stations of the Czech Republic measuring during a period of instrumental measurements (since 1771). For homogeneity testing there were used three relative homogeneity tests: Standard Normal Homogeneity Test by Alexandersson (1986), Bivariate Test of Maronna and Yohai (1978) and technique of Vincent (1996). Reference series were created by several ways to increase a number of results and thus to make the results more confident. All analysis in this paper are based on the author's software AnClim and ProcData (software for automatic processing of climatological data, homogenizing, adjusting, analyzing etc.). AnClim software is available at http://www.sci.muni.cz/ pest.
\end{abstract}

Key words: Temperature. Homogeneization. AnClim software. ProcData software. Czech Republic.

\footnotetext{
* Recibido: 17-3-2003 Aceptado: 10-6-2003
} 


\section{Introducción}

Parece razonable asumir que toda investigación sobre el cambio del clima debería realizarse con series de datos homogéneos, pero desafortunadamente las series de registros climáticos más prolongados están a menudo afectadas por factores no climáticos. Dichos factores se relacionan con la reubicación de los observatorios, con cambios en los procedimientos de registro de los datos, de los instrumentos etc., información que recibe el nombre de metadatos. Desgraciadamente los casos en que dichos metadatos son incompletos, no existen, o incluso se han perdido, son muy numerosos.

Por otra parte, existen numerosas técnicas estadísticas para detectar inhomogneidades en las series de datos climáticos. Las más empleadas en los últimos años se describen en Peterson et al. (1998), junto a los diversos planteamientos con los que se han estudiado estos problemas.

En la República Checa los problemas de homogeneidad de los datos climáticos solamente han sido solucionados ocasionalmente por el Instituto Hidrometeorológico (Czech Hydrometeorological Institute), de manera que esta labor se ha realizado hasta el presente de manera individual (por ejemplo Brázdil, 1996). El presente trabajo continua los esfuerzos de estas investigaciones y presenta los resultados y la experiencia adquirida en el proceso de homogeneizado de las series completas de temperatura (valores promedio mensuales) de la República Checa durante el período instrumental desde el año de 1771. Las inhomogeneidades detectadas y ajustadas en la presente investigación fueron solamente las detectadas como cambios simples. A partir de las series homogeneizadas se ha calculado la serie regional de temperaturas de la República Checa.

El proceso de homogeneización y análisis temporal se ha efectuado por medio del programa AnClim desarrollado por el autor (Štěpánek, 2003a) de libre disposición (http://www.sci.muni.cz/ pest). Para preparar las series de datos y analizar los resultados de dicho programa se ha empleado una segunda utilidad, denominada ProcData (Štěpánek 2003b).

\section{Bases de datos}

Las primeras medidas instrumentales climáticas de la República Checa comenzaron a mediados del siglo XVIII. Praga (observatorio Prague - Klementinum) posee la serie de registros continuos más prolongados de todo el país, desde 1771 hasta el pre- 
sente. Con posterioridad, el principal desarrollo de la red de observatorios comenzó de la mano de las sociedades meteorológicas durante el siglo XIX, para ser finalmente centralizado el servicio en el Instituto Central de Meteorología y Magnetismo Terrestre de Viena en 1848 (Augustin, 1885).

En el presente trabajo, las fuentes consultadas para estudiar las temperaturas medias del aire han sido los libros anuales (Yearbooks) del Instituto Central de Meteorología y Magnetismo Terrestre de Viena (1848-1856, 1864-1915), los Informes de la Comisión Meteorológica de la Sociedad de Ciencias Naturales de Brno (18811911), los libros anuales de las observaciones atmosféricas de las estaciones meteorológicas de Checoslovaquia (1916-1960), y los datos digitalizados desde 1961 hasta el presente del Servicio Hidrometeorológico de la República Checa (Czech Hydrometeorological Institute). Además de las citadas fuentes procedentes de estaciones climáticas, se han empleado en algunos casos diversas medidas de temperatura del aire procedentes de una densa red de observatorios de precipitaciones (sobre todo para realizar el relleno de datos ausentes en observatorios muy cercanos), así como otros datos procedentes de los archivos originales no publicados en los libros anuales.

Durante el proceso de adquisición de datos hubo que resolver distintos problemas, particularmente en épocas pasadas. Por ejemplo, en los registros anuales del antiguo Imperio Austríaco los valores de temperatura del aire se proporcionan en la escala Réaumur hasta 1870 , por lo que estos valores tuvieron que ser convertidos a grados Celsius. En otras ocasiones, con anterioridad a 1895 , el método de registro horario difería del presente $((\mathrm{t} 7+\mathrm{t} 14+\mathrm{t} 21+\mathrm{t} 21) / 4)$ por lo cual el valor promedio hubo de ser recalculado.

La evolución desde 1864 del número de observatorios del territorio de la actual República Checa se muestra en la Figura 1, donde se recogen únicamente los inclui-

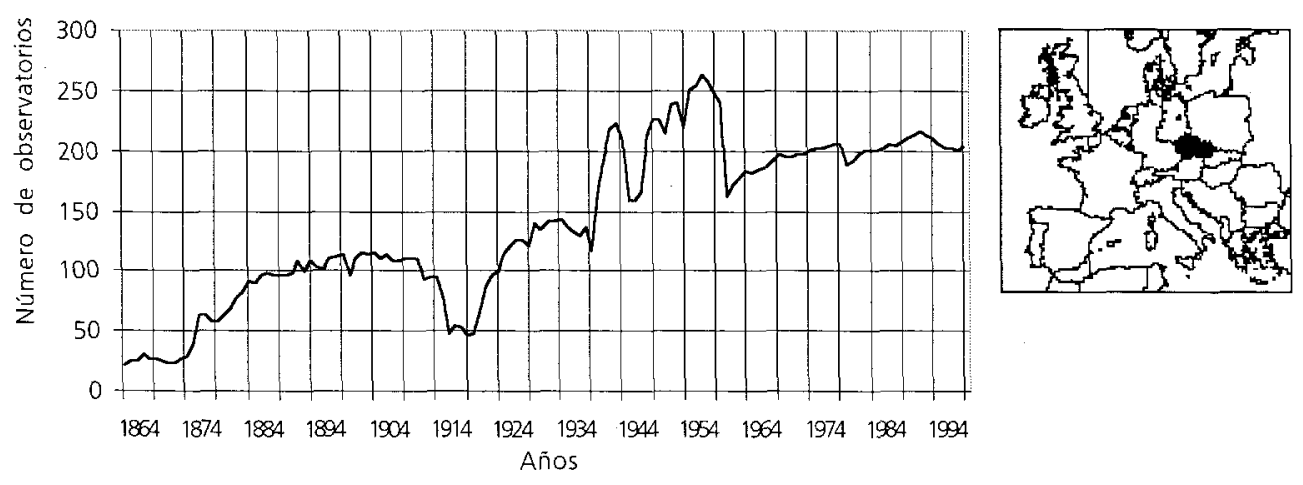

Figura 1. Evolución del número de observatorios climáticos en la República Checa (1864-2000) según los resúmenes anuales. 
dos en los resúmenes anuales. Los cambios que se observan deben su origen a las reorganizaciones de la red de observaciones, a los cambios de las instituciones responsables, las guerras etc.

Finalmente, para lograr un mayor grado de fiabilidad en los resultados sobre todo en los años iniciales de los registros, se han empleado diferentes series con registros prolongados procedentes de los estados vecinos (Austria, Alemania, Polonia y Eslovaquia). Tales series son sumamente importantes debido a que durante los primeros años no existen en el territorio de la República Checa suficientes mediciones para construir series de referencia.

\section{Procesamiento de datos}

Las pruebas de detección de inhomogeneidad, no obstante, deben ser observadas con cautela debido a su incertidumbre. Como ejemplo, en la Figura 2 se muestran los resultados obtenidos tras trabajar con cerca de 1000 series generadas aleatoriamente, con las propiedades de las series anuales de temperatura (por ejemplo varianza). En dichas series se introdujeron diferentes rupturas, con distintas magnitudes y en distintos momentos. La Figura 2 incluye una densa información. Permite concluir, por ejemplo, que la correcta detección de inhomogeneidades (error de estimación 0 años) con magnitudes inferiores a $0.5^{\circ} \mathrm{C}$ ocurre en menos de la mitad de los casos. Por su parte, la detección correcta de los cambios cuando estos son inferiores a $0.5^{\circ} \mathrm{C}$ es problemática. La presencia de valores porcentuales superiores a $100 \%$ se debe al proceso de análisis, detalles sobre dicho estudio se pueden consultar en Štěpánek (2003c).

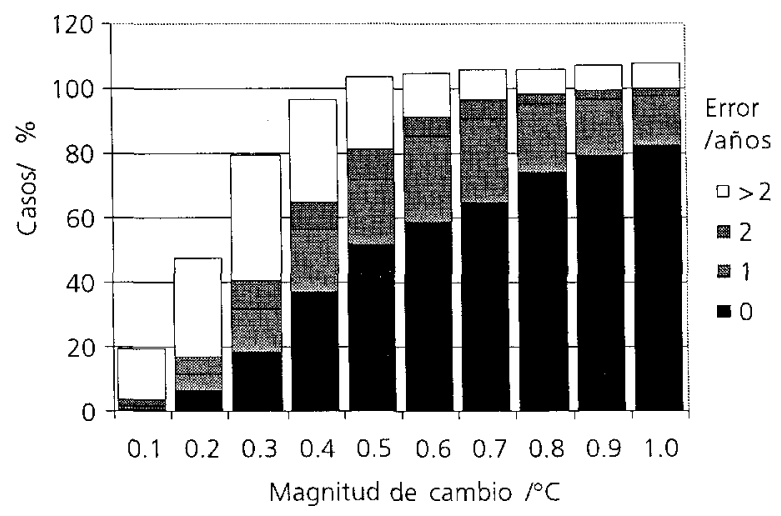

Figura 2. Porcentaje de casos detectados según distintas magnitudes de cambio introducidas artificialmente y error de la detección del año correcto. Extractado a partir de información obtenida de 950 series y SNHT, con $\alpha=0.05$. 
Esta es una de las razones fundamentales de que el método de análisis presentado en este trabajo incluya diferentes pruebas de inhomogeneidad, diferentes series de referencia etc, para incrementar el grado de confianza de la detección de inhomogeneidades. Un esquema general con los distintos pasos se muestra en la Figura 3.

El procesamiento de los datos incluye los siguientes pasos: la búsqueda, comprobación y corrección de valores extremos y datos sospechosos (quizá posibles errores), la creación de series de referencia mediante tres métodos diferentes, la comprobación de la homogeneidad de cada serie por medio de tres pruebas distintas, el ajuste de las inhomogeneidades detectadas y finalmente el relleno de los datos perdidos.

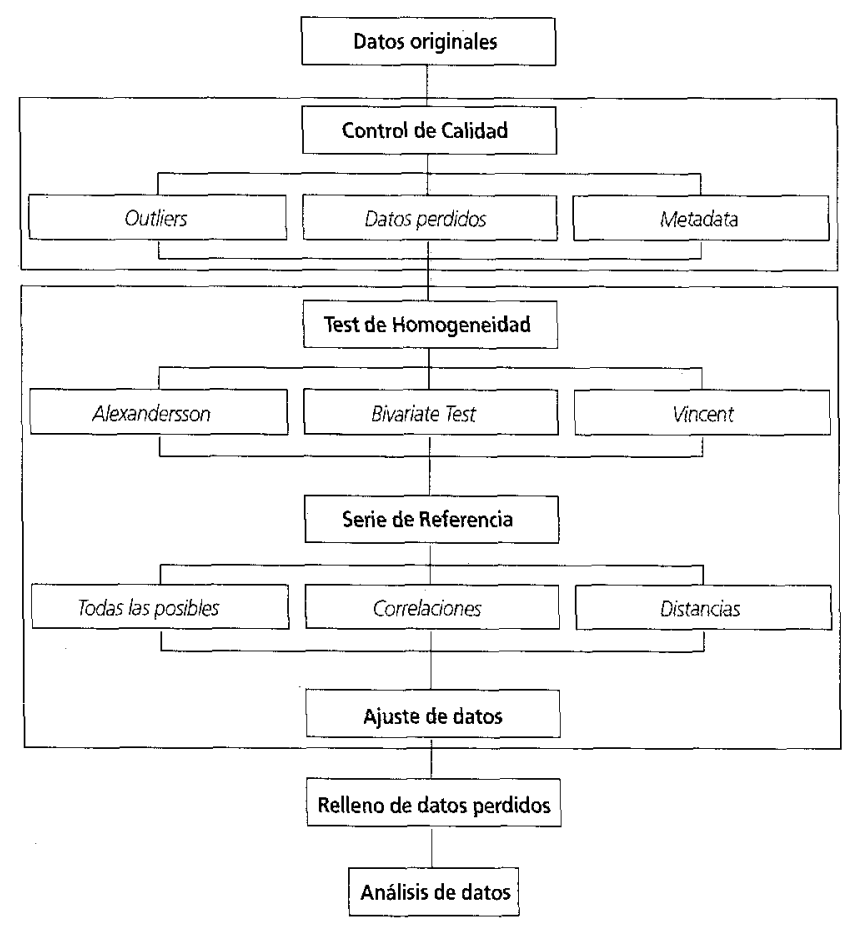

Figura 3. Esquema general del método de procesado de datos climáticos empleado en el presente estudio.

Como paso previo al estudio de homogeneidad de las series fue necesario comprobar la presencia de sucesos extremos y datos sospechosos. La detección se realizó en los libros de registro anual, y en caso de discrepancias en los propios archivos. Para detectar los datos calificados como extremos se definió un umbral a partir de 1.5 veces el rango intercuartílico; los datos sospechosos se consideraron en el caso de 3 
veces el citado rango. Si un dato fue considerado como extremo se dejó en la serie; en caso de estimar su carácter anómalo se eliminó de la serie y fue completado tras la homogeneización (véase 3.5). En ambos casos (valores extremos y datos anómalos) la detección se realizó sobre los datos de la serie de diferencias entre la serie candidata y la serie de referencia.

\subsection{Las pruebas de homogeneidad}

Una serie de datos climáticos es homogénea si "...sus variaciones solamente se han originado por las variaciones del tiempo y el clima" (Conrad y Pollak, 1950). Por otro lado la variedad de pruebas para detectar y ajustar inhomogeneidades en las series de datos climáticos es grande. Muchas de estas pruebas se basan en la denominada homogeneidad relativa. Así, una serie se dice que es "relativamente homogénea, respecto a una serie sincrónica de otro lugar, cuando la serie formada por las diferencias anuales en los valores de temperatura (razones en caso de la precipitación) constituye una serie aleatoria que satisface la ley del error" (Conrad and Pollak, 1950).

En el presente trabajo se emplea dicha inhomogeneidad relativa, lo que significa que en cada caso se compara la serie estudiada con una serie de referencia. La descripción de este procedimiento se detalla en 3.2 .

Las pruebas de homogeneidad se realizaron con el programa AnClim (Štěpánek 2003a) empleando los tres test siguientes, y nivel de significación $\alpha=0,05$ :

- Test de Alexandersson, en su versión original para detectar un solo cambio (SNHT for a single shift, Alexandersson 1986, 1995). La prueba se aplicó a las series mensuales, estacionales y anuales.

- El test bivariado de Maronna y Yohai (Maronna y Yohai 1978, Potter 1981), aplicado a las mismas series que el anterior.

- El método de Vincent, en su variante modelo 3 (Vincent, 1996). En este caso solamente aplicado a las series estacionales y anuales.

Las dos primeras técnicas se basan en la desviación acumulada sobre la media. La tercera es semejante al test propuesto por Easterling y Peterson (Easterling y Peterson 1995) y se fundamenta en una regresión lineal en dos fases.

En cada caso las series fueron tratadas en intervalos de longitud máxima de 40 años, pues las dos primeras pruebas solamente se emplearon en las versiones que detectan un solo cambio. Cuando los registros de las series fueron de mayor duración 
a 40 años, cada serie se dividió en tramos solapados entre si 10 años. Este solapamiento es importante pues la detección de inhomogeneidades es muy común en los años iniciales y finales de cada serie comprobada (véase Alexandersson 1995). Las series de referencia, a su vez, fueron calculadas para cada subserie.

La selección de periodos máximos de 40 años parece ser razonable pues series de menor longitud no serian tan aptas para un análisis estadístico, y por otra parte las series de mayor longitud muy frecuentemente contienen más de una inhomogeneidad. Por ejemplo, según Auer (2001), se puede suponer que una inhomogeneidad generalmente abarca intervalos no superiores a $30-40$ años.

\subsection{Las series de referencia}

El empleo de series de referencia supone aceptar un conjunto de requisitos, tales como que exista una elevada correlación con la serie candidata, que a su vez la serie de referencia sea homogénea, etc. En el caso de las temperaturas no existen problemas en cuanto a la correlación entre observatorios dentro del área de la República Checa. Para garantizar la homogeneidad de la serie de referencia se han empleado los promedios de diversos observatorios seleccionados. De este modo, los cambios posibles de las estaciones seleccionadas no deberían manifestarse en la serie de referencia final empleada para la homogeneización.

Finalmente para obtener un mayor grado de fiabilidad de los resultados de las distintas pruebas las series de referencia se calcularon por tres métodos distintos:

- Como promedio de todas las series disponibles.

- Como promedio de series seleccionadas a partir de sus correlaciones.

- Como promedios ponderados a partir de criterios de distancia.

Los tres tipos de series de referencia se han empleado porque producen valores diferentes y de este modo los resultados del proceso de homogeneización pueden ser analizados de modo más fiable. El primer tipo de series de referencia (promedio de todas las series disponibles), es el menos correlacionado con las series candidatas (aunque supera el valor de 0.9), pero tiene la ventaja de que elimina mejor inhomogeneidades en comparación con los otros dos métodos. En el segundo caso, la elevada correlación entre la serie candidata y la serie de referencia puede afectar y suprimir la aleatoriedad en la serie de diferencias o ratios empleada para el cálculo de inhomogeneidades; de este modo pudiera darse el caso de que el observatorio candidato y la serie de referencia compartiesen las mismas inhomogeneidades que que- 
darían anuladas (aunque este peligro se aminora empleando series de diferencias interanual, Alexandersson, 1995). En el tercer caso, las series de referencia calculadas por medio de las distancias con la serie candidata, se preserva el criterio de cercanía, aunque pudieran producirse condiciones climáticas distintas (diferentes alturas etc.).

\subsubsection{Las series de referencia a partir del promedio general}

Estas series de referencia fueron calculadas con los promedios anuales de todos los datos disponibles por medio de los valores convertidos en anomalías, de modo que los datos procedentes de diversas series fuesen comparables entre si. El procedimiento empleado fue el siguiente:

- Cada serie fue convertida en anomalías anuales, resultando una serie de media cero durante el período 1961-1990. El cálculo se realizó mensualmente.

- En las series sin mediciones durante el periodo de referencia, los promedios de estas series candidatas fueron comparados con los promedios de las series de referencia (seleccionadas entre las estaciones con mayor correlación) durante 30 años comunes. El cálculo se realizó mediante la comparación de los promedios de ambas series, estimando en su caso el valor de ajuste que debería aplicarse.

En la Figura 4 se muestra gráficamente el proceso seguido. Finalmente, una vez convertidos todos los valores anuales de las distintas series en anomalías respecto al periodo normal 1961-1990, se calculó la serie nacional de referencia de la República Checa, empleada como referencia general. Las series de observaciones con menos de 30 años no pudieron ser incluidas en este cálculo.

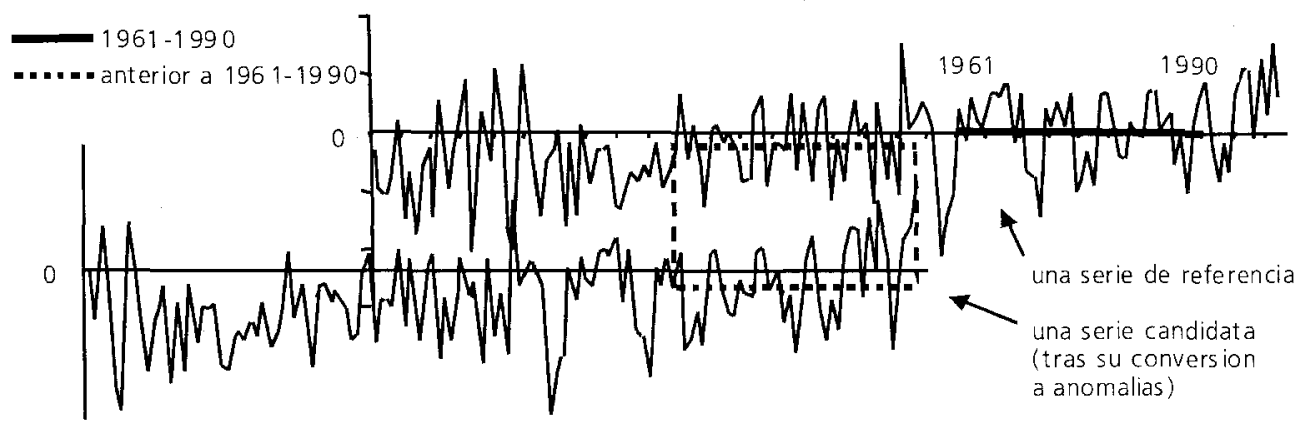

Figura 4. Ejemplo de conversión de una serie anterior a 1961-1990 en anomalías sobre el período citado. La serie de referencia (superior en la figura) y la serie candidata (inferior) al convertirse en anomalías sobre 1961-1990 pueden ser comparadas por tener el mismo promedio en algún periodo consecutivo de 30 años. 


\subsubsection{Series de referencia calculadas por medio de correlaciones}

En este caso cada serie de referencia se calculó como promedio de las medidas de aquellas estaciones más correlacionadas con la estación candidata correspondiente. Las correlaciones fueron calculadas sobre las series de diferencias interanuales, de manera que las posibles inhomogeneidades se manifestasen solamente en un solo valor. El promedio calculado fue la media simple, pues en el caso estudiado no existen diferencias reales con una media ponderada debido a las similitudes entre los valores de correlación de las estaciones y sus elevados valores. Las estaciones seleccionadas debían cumplir otros requisitos, por ejemplo una correlación mínima y no presentar excesivos valores perdidos. En caso contratio las series podrían contener demasiadas lagunas, y en consecuencia haber incluido numerosas inhomogeneidades.

Por último, se han empleado distintas estaciones con registros prolongados procedentes de fuera de los límites de la República Checa (hasta un total de 20 de los distintos países fronterizos). Estas series se emplearon sobre todo en el análisis de los periodos iniciales porque durante estos momentos el número de observatorios en el interior de la República Checa es muy escaso e insuficiente para generar una serie de referencia de calidad.

\subsubsection{Series de referencia calculadas según distancias}

Estas series de referencia se generaron de modo similar al anterior, seleccionando en este caso los observatorios según el criterio de distancia. En cada caso se emplearon aquellas estaciones más cercanas con cada estación candidata, y los valores de cada peso de ponderación se estimaron a partir del cuadrado del valor inverso de la distancia. Se estableció un límite de distancia, y no se aceptaron estaciones más alejadas de dicho límite. Solamente se aceptaron series con registros que cumplieran los requisitos de mínimas lagunas indicados anteriormente. No se impusieron otras condiciones (semejante altitud, por ejemplo) dadas las elevadas correlaciones de las temperaturas entre observatorios de la misma región.

Los detalles de los límites empleados en la República Checa se encuentran indicados en el punto 4 .

\subsection{Análisis de homogeneidad}

Se han aplicado diferentes pruebas de homogeneidad con distintas series de referencia a las series mensuales, estacionales y anuales. Como se ha indicado previamente, el objetivo fue ganar seguridad en los resultados parciales para garantizar que las correcciones tuviesen la mayor fiabilidad posible. 
Debido a la ausencia generalizada de metadatos, que hubieran podido informar de la existencia de posibles cambios, ante los resultados de las diversas pruebas estadísticas se establecieron distintos requisitos para aceptar las inhomogeneidades detectadas con mayor confianza y poder efectuar la corrección.

El primer criterio fue el número de inhomogeneidades en el mismo año. Sobre este particular se recuerda que el número total de resultados de cada serie supera el valor de 100 , acorde el número de test, de las series de referencia y el número de series mensuales, estacionales y anuales empleados. Para aceptar cada inhomogeneidad y realizar el ajuste, se establecieron dos umbrales sobre todas las posibles detecciones en cada observatorio, el primero cuando no existian metadatos y el segundo cuando dicha información existía. En segundo lugar se consideró el número de inhomogeneidades detectadas en grupos de años consecutivos, es decir: saltos que comenzando en un año determinado perdurasen al menos durante dos o más años. Otros criterios empleados para aceptar las inhomogeneidades fueron el análisis de su distribución estacional, los gráficos de diferencias con la serie de referencia etc.

En algunos casos las series fueron sumamente problemáticas para aceptar y, en consecuencia, efectuar el ajuste. Por ejemplo cuando se detectó un elevado número de inhomogeneidades pero no fue posible encontrar patrón alguno en el año de la detección. En estos casos las series (o sus subperiodos) fueron excluidas del análisis y posterior procesamiento.

\subsection{Ajuste de las series}

El ajuste de las inhomogeneidades detectadas y aceptadas se realizó con la serie de referencia calculada como promedio de los observatorios más correlacionados (véase 3.2.2). El valor de ajuste se estimó como la diferencia de los promedios del período comprendido \pm 20 años del año siguiente al del salto detectado. El periodo fue truncado en aquellos casos en que se detecto una nueva inhomogeneidad.

Las inhomogeneidades de los últimos cuatro años de cada serie no pudieron ser corregidas. Esta situación es relativamente común en la República Checa por el cambio debido a la automatización de los registros. En consecuencia estos tramos finales de las series con inhomogeneidades reales o aparentes fueron eliminados del análisis final.

Los ajustes en todo caso se aplicaron a las series mensuales. Cuando fue posible se estimó el inicio de las inhomogeneidades mensualmente. 


\subsection{Consideraciones finales del proceso de identificación de inhomogeneidades.}

El análisis presentado en sus sucesivos pasos fue aplicado iterativamente. Tras cada iteración se recalcularon de nuevo las series de referencia para cada tramo o subserie analizado. No se detectaron ulteriores inhomogeneidades tras la tercera iteración. El ajuste final de las inhomogeneidades se realizó sobre los datos originales con las correspondientes series de referencia construidas a partir de series homogeneizadas.

Una vez completado el análisis previo, los datos ausentes fueron rellenados por medio de regresiones lineales entre la serie candidata (variable dependiente) y la serie de referencia (variable independiente). Esta nueva serie de referencia fue creada en cada caso con las estaciones más correlacionadas y el modelo de regresión calculó los valores ausentes a partir de los valores del periodo \pm 20 en torno al dato perdido.

\section{Homogeneización de las series de la República Checa}

Con anterioridad al proceso descrito en la República Checa se disponía de 192 series de temperatura del aire no homogéneas. Durante el proceso de homogeneización fueron eliminados aquellos observatorios cuyas series resultaron poco fiables o no pudieron homogeneizarse. Otras series tuvieron que ser acortadas en los años finales por las razones expuestas, particularmente tras la automatización realizada desde 1997. Finalmente, la base de datos de temperaturas medias mensuales de la República Checa consta de 174 observatorios cuyas series son homogéneas.

En cada caso las series de referencia (véase 3.2 .2 y 3.2.3) se crearon a partir de cinco series (la construcción de series de referencia con más observatorios produjo los mismos resultados). El período común mínimo para calcular las correlaciones fue de 20 años, el valor aceptado de los coeficientes de correlación fue 0.9 (véase 3.2.2). El límite espacial para seleccionar observatorios cercanos fue de $100 \mathrm{~km}$ (véase 3.2 .3 ). Las estaciones seleccionadas no debían tener valores perdidos en promedio superiores a 1 dato cada 1.5 años. El criterio establecido para aceptar inhomogeneidades en ausencia de metadatos fue el $20 \%$ sobre el total de posibles inhomogeneidades. Cuando existieron metadatos el umbral se redujo a $15 \%$. 
Tabla 1. Resultados generales del proceso de homogeneización de las series de temperatura media de la República Checa.

\begin{tabular}{|c|c|c|}
\hline \multirow{2}{*}{ Características } & \multicolumn{2}{|c|}{ Datos } \\
\hline & Originales & Ajustados \\
\hline Observatorios & 192 & 174 \\
\hline Series ( 40 años de longitud) (*) & 348 & 307 \\
\hline Ajustes realizados & & 231 \\
\hline Series comprobadas & 40716 & 35919 \\
\hline Inhomoeneidades detectadas, $\mathrm{p}=0.05$ & 32445 & 13802 \\
\hline $\begin{array}{l}\text { \% de inbomogeneidades presentes respecto al } \\
\text { múmero de series analizadas }\end{array}$ & 79.7 & 38.4 \\
\hline
\end{tabular}

En la Tabla 1 se resumen los datos globales del proceso de reconstrucción. El elevado número de series analizadas se debe al hecho de haber trabajado en diferentes escalas temporales, al número de series de referencia empleadas y al número de pruebas de homogeneidad aplicadas. Tras la iteración 3 del proceso de identificación de inhomogeneidades, el número total de inhomogeneidades aceptadas fue de 231 que fueron corregidas. Con anterioridad a la homogeneización casi el $80 \%$ de las series (consideradas en tramos de 40 años de longitud) fueron identificadas como no homogéneas. Tras el ajuste de aquellas aceptadas como reales, el 40\% de las series continuó siendo no homogéneas.

La Figura 5 muestra el número de inhomogeneidades significativas $(a=0.05)$ antes y después del proceso de análisis. Las inhomogeneidades se detectaron principalmente en los meses de verano, siendo su número más del doble que el detectado en los meses de invierno. Tras el ajuste, el número de inhomogeneidades descendió notablemente sobre todo en verano, prácticamente la tercera parte, mientras que en invierno el descenso solamente fue la mitad del número original. Como se puede observar en las series finales aún persisten numerosas inhomogeneidades, hasta un $40 \%$ del total (Tabla 1). El ajuste de estas series es sumamente difícil de realizar debido a la incertidumbre de la estimación de los saltos sin otra información adicional proveniente de los metadatos (véase Figura 2)

La distribución de las magnitudes de los valores de las medianas de los ajustes se muestra en la Figura 6. Los valores más altos se corresponden con los meses de verano (en promedio $>0.5^{\circ} \mathrm{C}$ ), y los menores valores de corrección al invierno (pro- 
medio $>0.2^{\circ} \mathrm{C}$ ). El menor número de inhomogeneidades detectadas durante el invierno (Figura 5) y los inferiores valores de ajuste de estos saltos (Figura 6) se debe principalmente a que las temperaturas invernales están influenciadas por factores derivados de la circulación atmosférica. Por su parte, en los meses de verano la temperatura se relaciona con factores radiativos, espacialmente más dependientes y por tanto más variables. Estas circunstancias pueden apreciarse a su vez en los cambios de los valores de los coeficientes de correlación mensual entre las series candidatas y de referencia antes y después de realizar los ajustes (Figura 7).

Tal como se puede apreciar en la Figura 7 , tras el ajuste realizado los valores de correlación de cada serie con su serie de referencia aumentan notablemente en los meses de verano, mientras que en los meses de invierno tras el ajuste las correlaciones no se modifican del mismo modo, lo cual se debe al bajo valor de la corrección aplicada en estos meses.
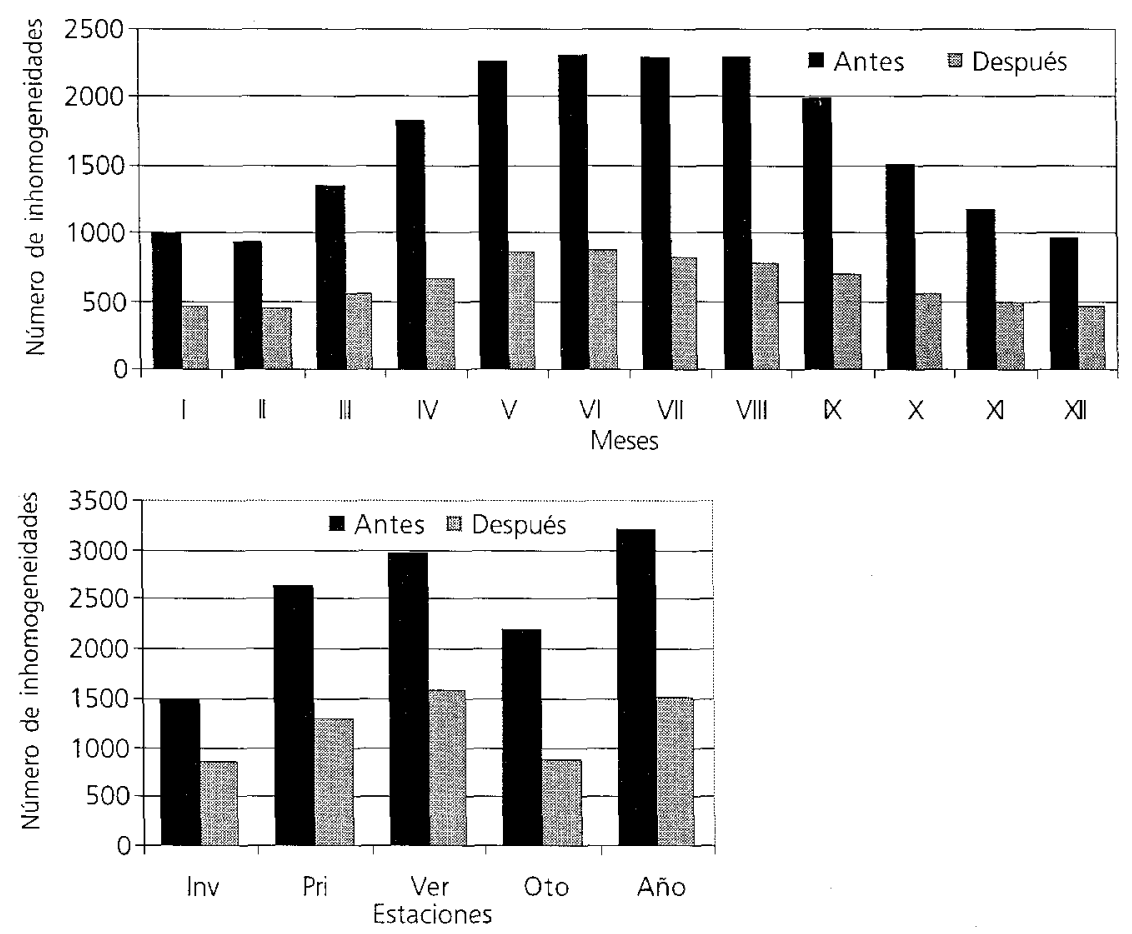

Figura 5. Número de inhomogeneidades significativas (mensuales, estacionales y anuales) detectadas antes y después del proceso de homogeneización. Número total de series comprobadas: antes de la corrección, en torno a 41.000 , después de la corrección, en torno a 36.000 series $(\alpha=0.05)$. 


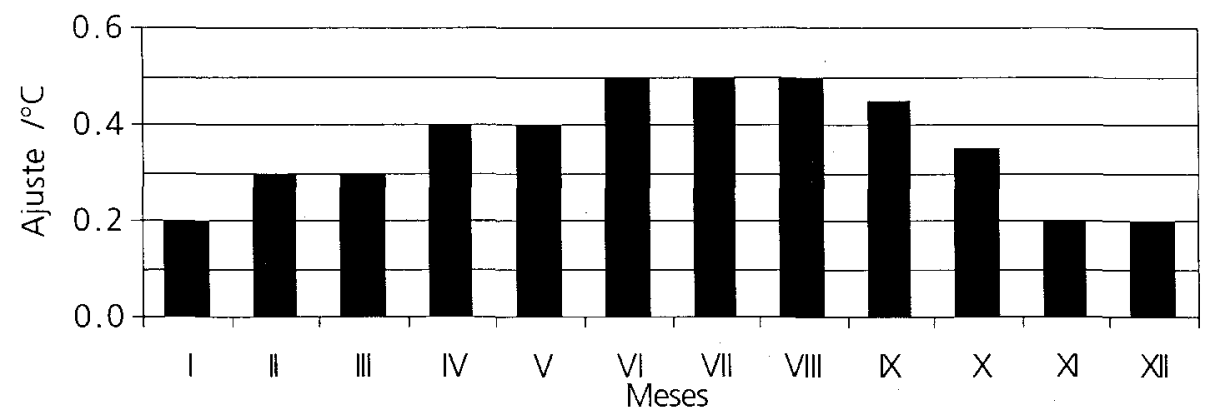

Figura 6. Valor de la mediana de las cantidades ajustadas en las series. Valores absolutos $\left({ }^{\circ} \mathrm{C}\right)$ Series ajustadas 231.

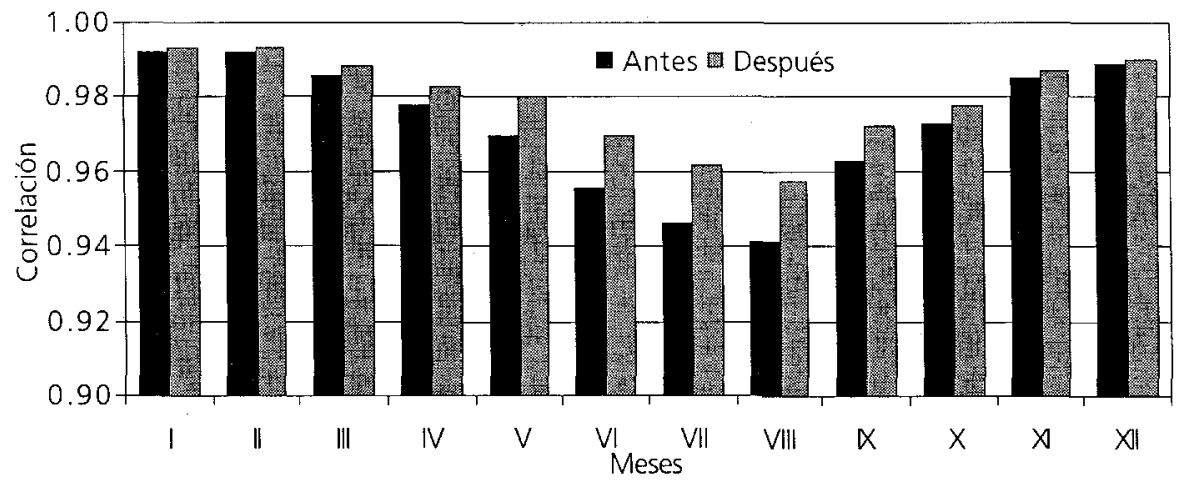

Figura 7. Mediana del coeficiente de correlación previo y posterior al homogeneizado entre las estaciones candidatas y de referencia (231 series ajustadas).

\section{Análisis de las series homogéneas}

Los resultados finales del proceso descrito han producido 174 estaciones que cumplen los criterios de calidad para realizar su análisis.

La Figura 8. muestra las disponibilidades de esta base de datos en cada año (compárese con la Figura 1). En promedio las series finales tienen 60 años de regis- 
tros (mediana 50 años), y la distancia mínima entre ellas es de $13 \mathrm{~km}$ (igual mediana). El número de observatorios con 20-39 años de registros es de 41, en 80 casos los registros abarcan entre 40-59 años, 32 son los observatorios con longitudes comprendidas entre 60-99 años y hay 21 series con más de 100 años de observaciones mensuales.

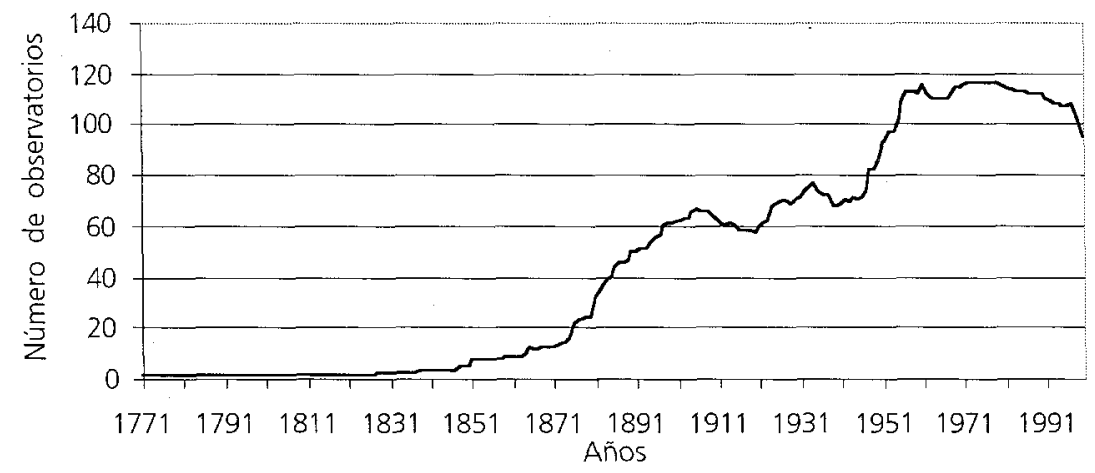

Figura 8. Número de observatorios analizados con series de registros termométricos homogéneos en la República Checa, desde el inicio del periodo instrumental.
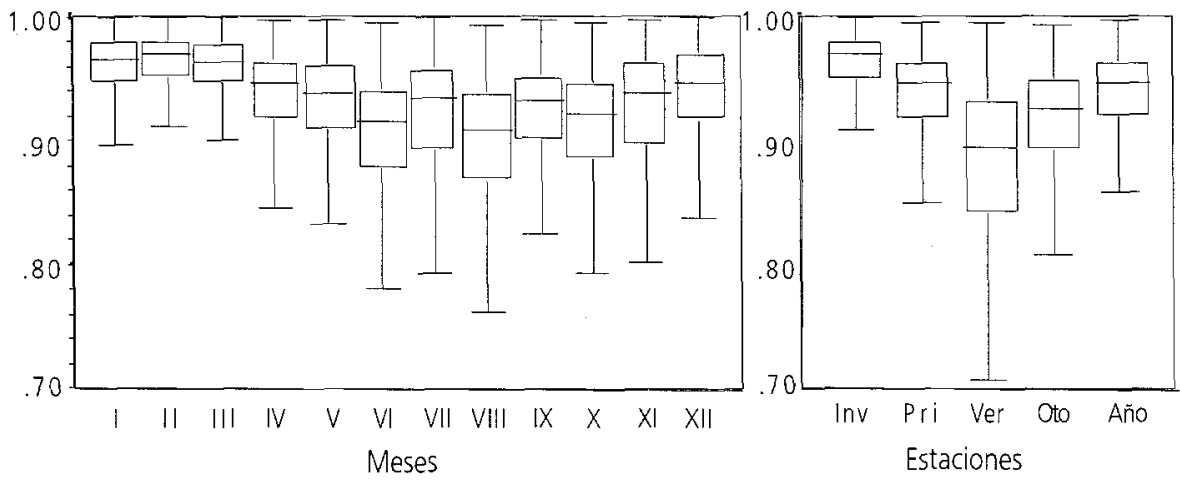

Figura 9. Coeficientes de correlación (box plot) mensual, estacional y anual entre todas las series. Cada caso representa 9187 valores.

Las variaciones entre los coeficientes de correlación mensuales, estacionales y anuales de las series homogeneizadas de temperaturas de la República Checa se pre- 
sentan en la Figura 9. Durante el invierno, cuando prevalecen las influencias de la circulación atmosférica sobre las temperaturas, se puede observar cómo la temperatura es muy semejante entre observatorios y los valores de correlación además son muy elevados. Durante el verano la variabilidad entre los valores de correlación entre todos los observatorios es superior.

Finalmente, siguiendo el método descrito en 3.2.1, se construyó la serie nacional de temperaturas de la República Checa, expresada como anomalías sobre el periodo de referencia 1961-1990. El período finalmente presentado abarca el intervalo 18482000. Para estimar el número de observatorios incluidos anualmente en el cálculo véase la Figura 8.

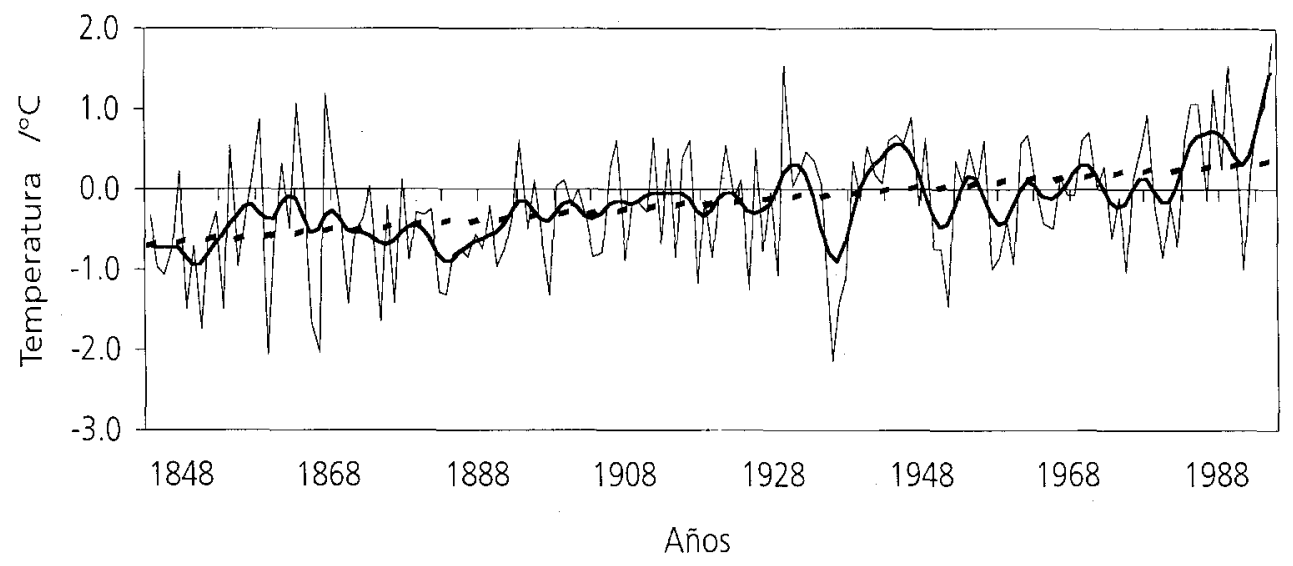

Figura 10. Temperaturas medias anuales de la República Checa 1848-2000, expresadas como anomalías sobre el período de referencia 1961-1990. Serie Nacional. Suavizado de 10 años con filtro de paso bajo de Gauss. La línea quebrada indica la tendencia lineal $1848-2000$.

Tabla 2. Tendencia lineal de la temperatura $\left({ }^{\circ} \mathrm{C} / 100\right.$ años) de la serie nacional de temperaturas de la República Checa, periodo 1848-200. En negrita valores significativos, $\alpha=0.05$.

\begin{tabular}{|lcccccccccccc|}
\hline Mes & I & II & III & IV & V & VI & VII & VIII & IX & X & XI & XII \\
Tendencia & $\mathbf{1 . 1 7}$ & 0.47 & $\mathbf{1 . 2 2}$ & $\mathbf{0 . 6 4}$ & $\mathbf{0 . 7 9}$ & 0.13 & 0.39 & $\mathbf{0 . 5 6}$ & 0.30 & 0.22 & $\mathbf{1 . 0 6}$ & $\mathbf{1 . 2 9}$ \\
\hline Estación & Inv. & Pr. & Ver. & Ot. & Año & & & & & & & \\
Tendencia & $\mathbf{0 . 9 6}$ & $\mathbf{0 . 8 8}$ & $\mathbf{0 . 3 6}$ & $\mathbf{0 . 5 2}$ & $\mathbf{0 . 6 9}$ & & & & & & & \\
& & & & & & & & & & & & \\
\hline
\end{tabular}


En la Tabla 2 se muestran los valores mensuales, estacionales y anuales de tendencia. En la Figura 10 se observa que los valores promedio anuales ofrecen una clara y significativa tendencia positiva durante el periodo $1848-2000$. Los valores presentados en la Tabla 2 indican que el incremento secular es de $0.69^{\circ} \mathrm{C} / 100$ años en los promedios anuales, y es consistente con las estimaciones realizadas sobre el calentamiento global de otras partes del continente y del planeta (Houghton et al., 2001).

El análisis realizado por medio de las técnicas MESA (Maximum Entropy Spectrum Analysis) permite observar que existen ciclos estadísticamente significativos $(?=0,05)$ con periodicidad de 7.8, 5.5 y 2.3 años (Figura 11).

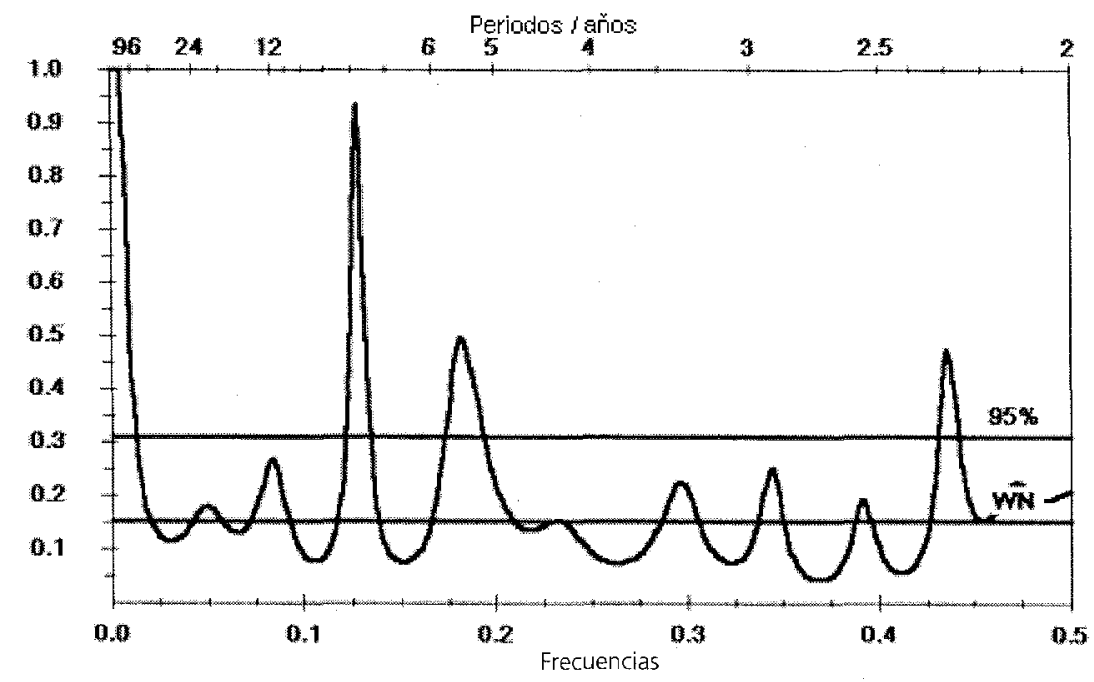

Figura 11. Análisis espectral (MESA) de los válores de temperatura media anual de la República Checa, 1848-2000, $m=30$.

\section{Conclusión}

En este trabajo se ha presentado el proceso de homogeneización de las temperaturas medias mensuales de la República Checa durante el periodo instrumental (desde 1771 hasta el presente). Uno de los objetivos principales fue desarrollar un método que en ausencia de metadatos (desafortunadamente la mayoría de los casos) lograse una mayor fiabilidad en la detección de inhomogeneidades. 
En el caso de la comprobación de inhomogeneidades relativas (la homogeneización por el método absoluto produce resultados menos fiables) una tarea fundamental fue la construcción de series de referencia. Las series se calcularon por tres procedimientos en cada caso. A su vez, cada pareja de series candidata y referencia fue comparada por medio de distintos métodos (tres pruebas de inhomogeneidad) y analizada mensual, estacional y anualmente.

Ante la incertidumbre generalizada en la detección de inhomogeneidades, el planteamiento sugerido en este trabajo presenta la ventaja de producir numerosos resultados en cada serie analizada lo cual permite aumentar la confianza en la detección. La decisión final para aceptar una inhomogeneidad como cierta se tomó considerando el número de inhomogeneidades detectadas, junto con las series de diferencias y otra información disponible; solamente entonces se aceptó una inhomogeneidad como indudable (de naturaleza física), incluso en ausencia de metadatos.

Incluso tras la homogeneización aún se mantiene una gran proporción de inhomogeneidades en las series analizadas (en torno al 40\% frente al $80 \%$ inicial), pero sin metadatos es muy arriesgado tomar decisiones al respecto y realizar la corrección de la inhomogeneidad detectada. Más aún, los test empleados para detectar inhomogeneidades anuales en más del $50 \%$ de los casos solamente son precisos cuando el cambio es superior a $0.5^{\circ} \mathrm{C}$. Por estas razones las tareas de investigación y desarrollo de otras pruebas para detectar inhomogeneidades deberían proseguir con el objeto de mejorar los resultados en relación con la investigación de series climáticas.

En el proceso de homogeneizado algunas partes de las series analizadas o incluso series completas tuvieron que ser excluidas debido a diversos problemas. Una cuestión que no ha sido solucionada es el problema de las inhomogeneidades localizadas al final de las series, cuyo ajuste no puede efectuarse porque su escasa longitud no permite estimar el valor de corrección. En el presente trabajo estas series fueron truncadas en el año de la detección. Tales casos son relativamente comunes en la República Checa durante los últimos años por el efecto de la automatización de la red de medidas. Sus efectos podrán analizarse cuando haya transcurrido un tiempo mínimo para garantizar medidas comparativas en la mayoría de los casos.

A partir de las series de temperatura del aire homogeneizadas se calculó la serie promedio nacional de la República Checa (1848-2000). En todas las estaciones y en los valores anuales existe una tendencia estadísticamente significativa. Los valores más elevados se corresponden con el invierno $\left(0.96^{\circ} \mathrm{C} / 100\right.$ años $)$, y los más bajos con el verano $\left(0.36^{\circ} \mathrm{C} / 100\right.$ años $)$. La serie anual muestra una tendencia de $0.69^{\circ} \mathrm{C}$ $/ 100$ años en el periodo analizado.

Tanto la serie homogeneizada nacional de temperaturas medias de la República Checa, como las diferentes series, serán objeto de análisis detallados en el futuro. 


\section{Agradecimientos}

El presente trabajo fue redactado con ocasión de una estancia de investigación del autor financiada por el proyecto REN2002-01023 (MCYT-FEDER). Especialmente se agradece la colaboración y facilidades aportadas por el prof. J.C.González Hidalgo (Dept. Geografía, Universidad Zaragoza). Se agradece a V. Kveton el haber proporcionado los datos digitalizados del periodo 1961-2000 empleados en la presente investigación.

\section{Bibliografía}

Alexandersson, A. (1986): A homogeneity test applied to precipitation data. J. Climatol., 6, p. 661-675.

Alexandersson, A. (1995): Homogenity Testing, Multiple Breaks and Trends. In: Proc. 6th Int. Meeting on Stat. Climatology, Galway, Irlanda, p. 439-441.

Auer, I., Böhm, R., Schöner, W. (2001): Austrian long-term climate 1767-2000. Multiple instrumental climate time series form Central Europe. Viena, Österreichische Beiträge zu Meteorologie und Geophysik, Heft 25.

Augustin, F. (1885): O potrebě zorganizovati meteorologická pozoroväni v Čechách (Sobre la necesidad de organizar las observaciones meteorológicas en Bohemia, en checo). Praga.

Bericht der meteorologischen Commission des naturforscbenden Vereines in Brünn 1881-1911. Brünn 1882-1917.

Brázdil, R. (1986): Variation of atmospheric precipitation in the C.S.S.R. with respect to precipitation changes in the European region. Universita J. E. Purkyně, Brno, 169 pp.
Brázdil, R., Štěpánek, P., Budíková, M. (1996): Homogenized air temperature series in Brno, 1891-1994. Zeszyty Naukowe Uniw. Jagiell., MCLXXXXVI, Prace Geograficzne, 102, p. $85-91$.

Conrad, V., Pollak, L.W. (1950): Mehods in Climatology. Harvard University Press, 459 pp.

Coufal, L., Langová, P., Míková, T. (1992): Meteorologická data na üzemi $\check{C} R$ za obdobi 1961-90. NKP ČSFR, sv. 8. ČHMÚ. Praga.

Easterling, D., R., Peterson, T., C. (1995): A new method for detecting undocumented discontinuities in climatological time series. Int. Journal of Climatology, 15, p. 369-377.

Houghton, J.T., Ding, Y., Griggs, D.J., Noguer, M., Van der Linden, P.J., Xiaosu, D. (2001): Climate Change 2001. The Scientific Basis. Cambridge: Cambridge University Press.

Jabrbücher der k. k. Zentral-Anstalt für Meteorologie und Erdmagnetismus 18481915. Viena.

Maronna, T., Yohai, V. J. (1978): A bivariate test for the detection of a systematic 
change in mean. J. Amer. Statist. Assoc., 73 , p: $640-645$.

Peterson, T.C. (1998): Homogeneity adjustments of in situ atmospheric climate data: a review. Int. J. Climatol. 18, p. 1493-1517.

Potter, K.W. (1981): Illustration of a New Test for Detecting a Shift in Mean in Precipitation Series. Mon. Wea. Rev., 109, p. 2040-2045.

Ročenka povětrnostnich pozoroväni meteorologických stanic 1916-1960 (Libro anual de observaciones meteorológicas de la red de observatorios meteorológicos de la República Checoslovaca, en checo). Praga p. 1934-1966.
Štěpánek P. (2003a): AnClim - Software for time series analysis. Dept. of Geography, Faculty of Natural Sciences, MU, Brno, 1.34 MB. http://www.sci.muni.cz/ pest.

Štěpánek P. (2003b): ProcData - Software for processing of climatological data. Dept. of Geography, Faculty of Natural Sciences, MU, Brno.

Štěpánek, P., (2003c): Air temperature variations in the Czech Republic in the period of instrumental measurements. Brno. Facultad de Ciencias Naturales, Depto. de Geografía, memoria de tesis. En preparación.

Vincent, L. A. (1998): A technique for the identification of inhomogeneities in Canadian temperature series. J. Climate, 11, p. 1094-1104. 